\title{
Recommandation concernant la répercussion des avantages obtenus dans le secteur ambulatoire lors de l'achat de médicaments prêts à l'emploi
}

Fritz Britt, sous-directeur, Office fédéral des assurances sociales

1 Recommandation concernant la promesse, l'acceptation et l'offre d'avantages matériels lies à des médicaments; obligation de répercuter les rabais obtenus.

2 Recommandation concernant la répercussion des rabais obtenus dans le domaine hospitalier lors de l'achat de médicaments prêts à l'emploi.

3 Le groupe de travail comprend des représentants des organismes suivants: SSPH, CDS, Comco, FMH, GSASA, H+, Swissmedic, Interpharma, SSIC, ACBIS, santésuisse, ASSGP, Office fédéral de la santé publique et Office fédéral des assurances sociales, ainsi que le Préposé fédéral à la surveillance des prix.
L'art. 33 de la loi sur les produits thérapeutiques (LPTh), entrée en vigueur le $1^{\text {er }}$ janvier 2002, règle d'une part la prescription et la remise de médicaments justifiés par une indication médicale et, d'autre part, en lien avec l'art. 56, al. 3, LAMal, la répercussion des avantages obtenus sur les agents payeurs.

La présente recommandation complète les recommandations de l'Office fédéral des assurances sociales (OFAS) qui vous ont été adressées le 21 décembre $2001^{1}$ et le 15 mars $2002^{2}$. Elle porte uniquement sur les avantages octroyés et obtenus dans le cadre des soins ambulatoires et non pas sur ceux octroyés ou obtenus dans le cadre des traitements hospitaliers (voir à ce sujet la recommandation de l'OFAS du 15 mars 2002); elle ne porte pas non plus sur les prestations matérielles.

S'appuyant sur les résultats du groupe de travail Rabais et bonifications, créé en novembre 2001 par l'OFAS et rassemblant les divers partenaires de la santé publique ${ }^{3}$, notre office formule, à propos de la répercussion des avantages pour les prestations relevant de l'assurance obligatoire des soins, les principes et les recommandations ci-dessous.

\section{Principes}

\section{Prescription par les médecins et remise} de médicaments par les pharmaciens Dans chaque cas particulier, la prescription de médicaments prêts à l'emploi doit être fondée uniquement sur des motifs médicaux.

Les avantages, notamment matériels, qui sont octroyés à un fournisseur de prestations adressant un patient à un confrère ou qui sont susceptibles d'inciter à mettre en œuvre des mesures thérapeutiques non indiquées sur le plan
LPTh: Art. 33 Promesse et acceptation d'avantages matériels

1 Il est interdit d'octroyer, d'offrir ou de promettre des avantages matériels aux personnes qui prescrivent ou remettent des médicaments ainsi qu'aux organisations qui emploient de telles personnes.

2 Il est interdit d'octroyer aux personnes qui prescrivent ou qui remettent des médicaments ainsi qu'aux organisations qui emploient de telles personnes de solliciter ou d'accepter des avantages matériels.

3 Sont admis:

a. les avantages matériels de valeur modeste et qui ont un rapport avec la pratique de la médecine ou de la pharmacie;

b. les rabais usuels dans le commerce ou justifiés économiquement qui se répercutent directement sur les prix.

$\rightarrow$ Dans le cadre des débats du Conseil des Etats sur la LPTh, il a été admis, par référence à la pratique pénale du Tribunal fédéral, que lors de la remise gratuite de biens il ne s'agit plus d'une valeur modeste au sens de la lettre à quand cette valeur excède le montant annuel de 300 francs par société et par médecin.

médical ou pharmaceutique tombent sous l'effet de l'art. 33, al. 1 ou 2 LPTh. L'octroi d'avantages à des fournisseurs de prestations dans le but de les influencer afin qu'ils remettent ou prescrivent des médicaments dont les indications ne sont pas uniquement médicales est interdit en vertu de l'art. 33 LPTh. 
On part de l'idée que le comportement des fournisseurs de prestations en matière de prescription et de remise des médicaments peut être influencé quand:

- la part relative à la distribution (cf. art. 67, al. $1^{\text {bis }}$ et $1^{\text {quater }}$ OAMal, art. 35a OPAS) dans la chaîne de distribution augmente pour les médicaments prescrits par un médecin, sans que cette augmentation s'explique par des rabais usuels dans le commerce ou justifiés économiquement;

- un membre de la chaîne de distribution remet gratuitement des biens au fournisseur de prestations;

- les avantages octroyés ou acceptés ne figurent ni sur le bon de livraison ni sur la facture et ils ne sont pas comptabilisés selon les règles comptables habituelles;

- le prix d'achat du fournisseur de prestations est inférieur au prix de fabrique, sans qu'il soit prouvé qu'il y a un rabais usuel dans le commerce ou justifié économiquement;

- dans le cadre de la livraison de médicaments par les fabricants ou les distributeurs, des ressources (personnes, appareils ou objets) sont mises à disposition du fournisseur de prestations, alors qu'elles ne sont pas indispensables à la remise ou à l'utilisation des médicaments en question;

- les fabricants ou les distributeurs proposent des rémunérations excessives pour la réalisation d'études.

\section{Les rabais justifiés économiquement au sens de I'art. 33 LPTh sont}

- les conditions de paiement particulières (escompte pour paiement anticipé, procédure de commande simplifiée, notamment par Internet, etc.);

- les abattements pour besoin logistique réduit du côté du vendeur (livraisons en gros ou moins fréquentes, stockage chez l'acheteur);

- l'indemnisation d'autres services fournis par l'acheteur (préparation des données, standardisation du processus, etc.).

\section{Les rabais usuels dans le commerce au sens de I'art. 33 LPTh sont}

des avantages qui, pour certains produits ou groupes de produits, ont été octroyés dans des cas particuliers sur un temps assez long, vont au-delà des rabais justifiés économiquement et ne sont pas contraires à l'art. 33 LPTh. Certains avantages octroyés jusqu'à présent ne sont cependant pas autorisés sur la base de l'art. 33 LPTh.

\section{LAMal: Art. 56 Caractère économique} des prestations

1 Le fournisseur de prestations doit limiter ses prestations à la mesure exigée par l'intérêt de l'assuré et le but du traitement.

2 La rémunération des prestations qui dépassent cette limite peut être refusée. Le fournisseur de prestations peut être tenu de restituer les sommes reçues à tort au sens de la présente loi. Ont qualité pour demander la restitution:

a. l'assuré ou, conformément à l'article 89, 39e alinéa, l'assureur dans le système du tiers garant (art. 42, $1^{\mathrm{er}}$ al.)

b. l'assureur dans le système du tiers payant (art. $42,2^{\mathrm{e}}$ al.).

3 Le fournisseur de prestations doit répercuter sur le débiteur de la rémunération les avantages directs ou indirects qu'il perçoit:

a. d'un autre fournisseur de prestations agissant sur son mandat;

b. de personnes ou d'institutions qui fournissent des médicaments ou des moyens et appareils diagnostiques ou thérapeutiques.

4 Si le fournisseur de prestations ne répercute pas cet avantage, l'assuré ou l'assureur peut en exiger la restitution.

5 Les fournisseurs de prestations et les assureurs prévoient dans les conventions tarifaires des mesures destinées à garantir le caractère économique des prestations. Ils veillent en particulier à éviter une réitération inutile d'actes diagnostiques lorsqu'un assuré consulte plusieurs fournisseurs de prestations.

\section{La répercussion des avantages au sens de l'art. 56 LAMal}

Les avantages obtenus lors de l'achat de médicaments prêts à l'emploi figurant dans la liste des spécialités doivent être répercutés dans la mesure où ils ne constituent pas la rémunération d'une contre-prestation usuelle dans le commerce ou justifiée économiquement (prise en charge des frais de stockage, etc.). Il y a répercussion d'un avantage, par exemple, quand celui-ci a été pris en compte, sous la forme d'une réduction de prix, par tous les maillons de la chaîne de distri- 
bution lors de la fixation du prix de vente ou bien qu'il a été répercuté sur l'assureur compétent conformément à un accord (tarifaire ou administratif) passé entre ce dernier et le fournisseur de prestations.

\section{Délimitation entre secteur hospitalier et secteur ambulatoire pour la répercussion des avantages sur les médicaments}

La répercussion des avantages sur les emballages de médicaments qui sont prescrits dans le cadre d'une hospitalisation ou sur les emballages entamés qui sont remis à un patient à sa sortie d'un établissement hospitalier est régie par les directives concernant le secteur hospitalier (cf. recommandation de l'OFAS du 15 mars 2002). Dans tous les autres cas, elle est régie par les recommandations ci-après pour le secteur ambulatoire.

\section{Définition du terme " grossiste »}

Est considérée comme grossiste toute personne physique ou morale qui possède une autorisation de faire le commerce en gros, délivrée par l'Institut suisse des produits thérapeutiques, Swissmedic, et qui remet des médicaments à des personnes habilitées à en faire le commerce, à les préparer, à les remettre ou à les utiliser à titre professionnel (art. 2, let. e, Ordonnance sur les autorisations dans le domaine des médicaments, OAMéd; voir aussi art. 28 et 29 LPTh et art. 7 à 11 OAMéd).

\section{Recommandations}

\section{Aux personnes autres que les fournisseurs de prestations}

\section{Fabricants/importateurs}

Il est recommandé de:

- comptabiliser selon les règles comptables habituelles (rabais, avantages, bonifications) les rabais/avantages octroyés et les indiquer sur le bon de livraison, la facture et l'avis de crédit pour chaque acheteur/client;

- établir, à la demande des acheteurs/clients, un relevé client sur les rabais/avantages/ bonifications octroyés;

- délivrer des échantillons uniquement sur demande écrite et établir les documents correspondants nécessaires (cf. art. 10, al. 1 et 5, Ordonnance sur la publicité pour les médicaments, OPMéd);

- adresser à l'OFAS une demande de baisse du prix maximum indiqué dans la liste des spécialités quand le prix de vente réalisé est régulièrement inférieur au prix de fabrique officiel et que le rabais octroyé ne correspond à aucune contre-prestation économique appropriée.

Les conditions d'une répercussion correcte par les acheteurs/clients des rabais et avantages obtenus au sens de l'art. 56 LAMal sont remplies du côté de la firme pharmaceutique et de l'importateur si les rabais/avantages/bonifications octroyés sont correctement comptabilisés (principe brut) et si une attestation est établie sur demande pour chaque client.

\section{Grossistes}

Il est recommandé de:

- communiquer toutes les conditions de rabais et de livraison, ainsi que les listes de prix;

- établir, à la demande des acheteurs/clients, un relevé client sur les rabais/avantages/ bonifications octroyés;

- adresser à l'OFAS une demande de baisse du prix maximum indiqué dans la liste des spécialités quand le prix d'achat réalisé est régulièrement inférieur au prix de fabrique officiel et que le rabais octroyé ne correspond à aucune contre-prestation économique appropriée.

Les conditions d'une répercussion correcte par les acheteurs/clients des rabais et avantages obtenus au sens de l'art. 56 LAMal sont remplies du côté du grossiste si les rabais/avantages/bonifications octroyés sont correctement comptabilisés et répercutés et si une attestation est établie sur demande pour chaque client.

\section{Aux fournisseurs de prestations}

(secteur ambulatoire des hôpitaux, pharmacies, médecins propharmaciens)

La facture établie à l'intention du débiteur de la prestation (tiers garant: personne assurée; tiers payant: assureur) doit être détaillée et compréhensible, de façon qu'il soit possible de vérifier le calcul de la rémunération et le caractère économique de la prestation (cf. art. 42, al. 3, LAMal).

Pour des livraisons régulières de médicaments à d'autres fournisseurs de prestations (distribution horizontale), il est renvoyé aux recommandations destinées aux grossistes (voir plus haut).

\section{Recommandations communes}

Il est recommandé de:

- répercuter sur la clientèle, sous forme de réduction sur les prix d'emballage, les rabais obtenus qui ne sont pas usuels dans le commerce ou justifiés économiquement; 
- indiquer pour chaque client les rabais et les avantages octroyés lors de la vente d'un médicament, de la manière suivante:

- dans le système du tiers garant, sur la facture (bon de caisse) et (en plus) conformément aux dispositions d'un éventuel accord tarifaire ou administratif passé avec l'assureur,

- dans le système du tiers payant, sur la facture adressée à l'assureur (cf. art. 42, al. 3, LAMal: copie à l'assuré) et conformément aux dispositions d'un éventuel accord tarifaire ou administratif passé avec l'assureur;

- faire parvenir à l'OFAS une documentation destinée à la Commission fédérale des médicaments (CFM), par l'intermédiaire de l'association de la branche concernée (H+, GSASA, $\mathrm{SSPH}, \mathrm{FMH})$, quand le prix de vente est régulièrement et systématiquement inférieur au prix de fabrique et que le rabais octroyé n'est pas usuel dans le commerce ou justifié économiquement;

- indiquer sur les emballages de médicaments le prix effectif à payer d'une manière visible et lisible (voir à ce propos les dispositions de l'ordonnance sur l'indication des prix, OIP, ainsi que la feuille d'information du seco du $1^{\text {er }}$ octobre 2001 sur l'obligation d'affichage du prix des médicaments);

- établir les documents relatifs aux opérations journalières en matière d'achat et de vente de médicaments, c'est-à-dire enregistrer, selon les règles comptables habituelles, les prix coûtants, les rabais/avantages/bonifications obtenus et octroyés et les prix de vente nets, et les indiquer pour chaque client (prix de vente net, prix de vente brut non compris les rabais/avantages).

Les conditions d'une répercussion correcte sur les assurés des rabais et des avantages au sens de l'art. 56 LAMal sont remplies du côté du fournisseur de prestations si les rabais/avantages/ bonifications octroyés sont correctement comptabilisés et répercutés sur les débiteurs de prestations et qu'une attestation est établie.

\section{Médecins prescripteurs}

Les prescriptions cantonales règlent les conditions (urgences par exemple) de la remise et du stockage de médicaments par les médecins.

Il est recommandé à ces derniers de ne pas travailler avec des pharmacies ou d'autres entreprises qui commercialisent des médicaments (pharmacies vendant des médicaments par correspondance ou par Internet, notamment), car celles-ci risquent d'influer sur leur comportement en matière de prescription (voir à ce propos Principes, al. 2, p. 2) quand cet arrangement permet de contourner l'interdiction de remise des médicaments ou qu'il est lié à des systèmes de participation.

Systèmes kick-back/Systèmes de participation Sont considérés comme des systèmes de participation ou systèmes kick-back des systèmes qui, lors de la distribution ou de la remise de médicaments, récompensent l'augmentation du chiffre d'affaires ou des quantités par des sommes versées en retour aux partenaires commerciaux. Il peut s'agir de rémunérations sous diverses formes: ristournes sur le chiffre d'affaires annuel, octroi d'actions d'entreprises à des prix préférentiels ou d'une part des bénéfices, livraisons de médicaments à prix réduit ou gratuites, fourniture par des tiers de prestations à prix réduit ou gratuites (prestations de laboratoire, voyages pour conférences ou congrès, équipement pour le cabinet ou la pharmacie avec garanties à des conditions préférentielles, livres, logiciels en rapport avec l'activité, matériel en promotion, etc.), financement d'études cliniques, postes de médecin assistant, etc. L'OFAS élaborera une recommandation séparée pour le domaine des prestations matérielles.

Il est recommandé de répercuter sur les partenaires commerciaux ou les patients les avantages mentionnés ci-dessus sous forme de réductions sur les prix des médicaments.

\section{Répercussion d'avantages ne pouvant être classés dans une catégorie particulière}

Si les avantages obtenus par un membre de la chaîne de distribution des médicaments ne peuvent pas ou ne peuvent plus (par exemple dans le cas d'avantages obtenus après coup) être mis en rapport avec les différents produits et donc classés dans une catégorie particulière, la répercussion sur les agents payeurs se fait dès que les avantages obtenus ont été versés sur le compte de la Regiobank Solothurn spécialement ouvert à cet effet par l'institution commune LAMal ( $n^{\circ} 16$ 0.009.518.04, médecins).

Il en est de même pour les avantages obtenus lors de l'achat d'autres types de produits (sur les produits en vente libre lors de l'achat de produits de la LS, par exemple). Il est recommandé de comptabiliser aussi ces avantages selon le principe du produit brut. 


\section{Codes de bonne conduite}

Afin de garantir la répercussion des avantages sur les débiteurs, l'OFAS recommande aux divers acteurs d'adopter un code de bonne conduite. Ce code doit rendre transparent leur comportement commercial en matière de promesses, d'acceptations et de répercussions de rabais ainsi que d'avantages matériels (voir aussi la recommandation de l'OFAS du 21 décembre 2001).

Dans le cadre de ce code de bonne conduite, l'OFAS recommande de traiter notamment les points ci-dessous.
- Partie générale:

- objectifs;

- champ d'application.

- Partie spécifique:

- documentation, transparence, communication;

- achat (organisation, instances de décision, indépendance de l'achat et des instances de décision, critères, principes);

- rabais et autres avantages:

- définition, répercussion: sponsoring et actions publicitaires, études cliniques, utilisation-type, primes, autres avantages matériels,

- façon de procéder avec les avantages que l'on peut obtenir en associant délibérément divers segments de produits (produits LS et produits en vente libre).

- Surveillance et sanctions.

- Rapport final. 